\title{
Indirect Dark Matter Searches with the MAGIC Telescopes
}

\author{
Jelena ALEKSIĆ* on behalf of the MAGIC Collaboration \\ Institut de Física d'Altes Energies, E-08193 Bellatera, Spain \\ E-mail: jelena@ifae.es
}

\begin{abstract}
MAGIC is a ground-based system of two, $17 \mathrm{~m}$ diameter Imaging Atmospheric Cherenkov Telescopes located in the Canary island of La Palma. MAGIC-I has been operational since 2004 and it already achieved the lowest energy threshold among the current generation of Cherenkov Telescopes. In 2009 it was joined by MAGIC-II, and together, in the stereoscopic mode, they allow for observations of substantially improved sensitivity, lower energy threshold and better energy and angular resolution.

The search for dark matter with MAGIC consists of looking for the signatures of very-high energy gamma-rays that originate from the annihilation of hypothetical dark matter particles. The gamma-ray spectrum resulting from that process should bear distinctive features (like spectral line or cutoff), correlated to the dark matter particle mass, which, according to some models, in the case of weakly interacting massive particles, should be in the very-high energy range and, therefore, detectable by the Imaging Atmospheric Cherenkov Telescopes. However, identification of these features, though they are universal, is a challenge, since the potential dark matter signal usually remains invisible under the dominant gamma-radiation from conventional astrophysical sources. For this reason, we direct our search with MAGIC to the objects with large amounts of dark matter, like the Galactic Center, the dwarf spheroidal galaxies and galaxy clusters.

Here we report on the indirect dark matter searches performed with MAGIC and discuss how these results can be used to set constrains on dark matter parameter space of certain supersymmetric models.
\end{abstract}

The 2011 Europhysics Conference on High Energy Physics-HEP 2011,

July 21-27, 2011

Grenoble, Rhône-Alpes France

\footnotetext{
* Speaker.
} 


\section{Introduction}

Despite strong efforts over the last decades, the nature of dark matter (DM) still remains one of the greatest mysteries of modern Physics. Observational evidence favor the scenarios where DM consists of weakly interacting, massive particles (WIMPs), of non-baryonic origin and produced thermally in the earliest stages of the Universe [1]. Although assumed stable over long periods of time, these WIMPs should be able to self-annihilate, producing Standard Model particles (like photons, leptons and hadrons), that we might detect from Earth. Supersymmetry (SUSY) provides a natural candidate for the DM WIMP - the lightest SUSY particle neutralino.

Given the natural mass range for the SUSY WIMPs (between a few $\mathrm{GeV}$ and a few TeV) [2], it may be possible to find, via very energetic photons, some signatures of DM annihilation in the energy range of Imaging Atmospheric Cherenkov Telescopes (IACTs). The typical annihilation gamma-ray spectrum is predicted to be continuous and featureless, due to the gammas mainly being produced from pion decay and final state radiation of charged particles. Nevertheless, some distinctive features could be present, like the monochromatic line, a cutoff or a spectral hardening due to the internal bremsstrahlung, all dependent on the mass of the DM particle.

\section{Candidates for Indirect Dark Matter Searches}

Photons produced in the DM annihilation are expected to reach the Earth with a flux that is determined by two independent components: one, coming from the particle physics, and the other, related to the astrophysics. The particle physics term is same for all DM regions - it is defined by the DM model and basically tells us how many gammas are produced above a certain energy threshold per WIMP annihilation, where in the spectra and which features to expect, etc. On the other hand, the astrophysical term is source dependent - it is determined by the DM density profile of a specific object, as well as its distance.

Therefore, when it comes to DM searches with IACTs, the regions and sources suitable for observations are those that are relatively close by, that have high concentration of DM, and that are (as much as possible) free from the gamma-ray emission of conventional astrophysical origin. Among such candidates are the Galactic Center (GC), dwarf Spheroidal Galaxies (dSphs), Galaxy Clusters and DM clumps that might exist in our Galaxy. Results of observations of some of these objects by the MAGIC Telescopes are presented in the following section.

\section{MAGIC Observations}

MAGIC has been observing the gamma-ray sky from the Canary island of La Palma $\left(28.8^{\circ} \mathrm{N}\right.$, $17.8^{\circ} \mathrm{W}, 2200 \mathrm{~m}$ a.s.1.) since 2004. In 2009 it became a stereoscopic system, therefore increasing the sensitivity of the observations, lowering the energy threshold down to $50 \mathrm{GeV}$ and improving the energy and angular resolution [3].

Over the years, MAGIC has observed several DM candidates, however, none of the cases resulted in a detection. The flux upper limits (ULs) were calculated, above a certain energy threshold, for every observation and then used to probe the parameter space of mSUGRA [4]. 


\subsection{The Galactic Center Observations}

The GC is the most obvious choice for DM searches: it is very close and the flux expected from DM annihilation is huge. However, the region hosts many astrophysical objects, whose gamma-ray emissions 'drown' any possible signals of DM origin.

MAGIC-I observed the GC for $\sim 24 \mathrm{~h}$ during 2004 and 2005 [5]. Given that the source culminates at about $58^{\circ}$ zenith angle at the telescope position, the energy threshold achieved in the analysis could not be lower than $1 \mathrm{TeV}$. A point-like emission was detected, however, it spatially coincides with Sgr A*. Possible DM explanation of the measured spectrum would have to involve a very massive neutralino (mass of order 10-20 TeV), and a boost factor of 2-3 orders of magnitude.

\subsection{Dwarf Spheroidal Galaxies Observations}

dSphs, the satellite galaxies of the Milky Way, have been identified as excellent candidates for indirect DM searches: their ratio between the mass inferred from gravitational effects and the mass inferred from the luminosity $(M / L)$ can reach values from 10 up to few $1000 M_{\odot} / L_{\odot}$; they are relatively close and they are expected to be free of astrophysical objects emitting at very-high energies (VHE).

Draco Observations Draco is one of the nine 'classical' dSphs, located $82 \mathrm{kpc}$ away from us, with $M / L$ estimated to $\sim 200 M_{\odot} / L_{\odot}$. Draco was observed with MAGIC-I for $7.8 \mathrm{~h}$ in 2007 [6]. The flux UL of $1.1 \times 10^{-11} \mathrm{~cm}^{-2} \mathrm{~s}^{-1}$ was found, for energies above $140 \mathrm{GeV}$, assuming a generic annihilation spectrum without a cutoff. Experimental values were compared to the ULs calculated for several mSUGRA benchmark models, but results showed that MAGIC Draco observations are an $\mathscr{O}\left(10^{3}-10^{9}\right)$ away from constraining the mSUGRA parameter space.

Willman 1 Observations Willman 1 was recently discovered as an ultra-faint dSph, located 38 kpc from us, with $M / L \sim 500-700 M_{\odot} / L_{\odot}$. Willman 1 was observed by MAGIC-I in 2008 for 15.5 $\mathrm{h}$ [7]. No significant gamma-ray emission was found above $100 \mathrm{GeV}$, corresponding to $2 \sigma$ integral ULs of order of $10^{-11} \mathrm{~cm}^{-2} \mathrm{~s}^{-1}$. Assuming the NFW profile of the DM halo, flux was calculated for four different mSUGRA modified benchmark models. ULs on the Willman 1 flux measured by MAGIC-I were still $\mathscr{O}\left(10^{3}-10^{5}\right)$ far from putting constraints on the considered DM models.

Segue 1 Observations Segue 1 is an ultra-faint dwarf located $23 \mathrm{kpc}$ away, with $M / L \sim 1320$ $3400 M_{\odot} / L_{\odot}$, making it the most DM dominated object known so far. The Segue 1 observations were carried out with MAGIC-I in late 2008 - early 2009, for a total of 29.4 h [8]. No significant gamma-ray signal was found above the background. Integral ULs are of order $\sim 1 \times 10^{-11}$ $\mathrm{cm}^{-2} \mathrm{~s}^{-1}$ (above $100 \mathrm{GeV}$ ) and $\sim 2 \times 10^{-12} \mathrm{~cm}^{-2} \mathrm{~s}^{-1}$ (above $200 \mathrm{GeV}$ ). Assuming the Einasto profile, for over $5 \times 10^{6}$ different mSUGRA models, the expected gamma-ray flux was calculated and compared to the corresponding ULs obtained from the observations. Results showed that the models for which the relic density is in agreement with the WMAP value are still at least a factor $\sim 600$ away from being detected.

\subsection{Galaxy Clusters}

Galaxy Clusters are the largest and most massive gravitationally bound systems in the Universe, with radii of the order of Mpc and masses around $10^{14}-10^{15} \mathrm{M}_{\odot}$. The amount of DM in 
Galaxy Clusters is predicted to be huge ( $80 \%$ of the total mass), however, the identification of the potential DM signal is made difficult by emissions from individual galaxies and cosmic rays.

Perseus The Perseus Galaxy Cluster was chosen for observations as a good DM candidate; nevertheless, the observations carried out with MAGIC-I in 2008, for 24.8 hours [9] resulted in no excess over the background. The integral flux ULs above $100 \mathrm{GeV}$ were calculated to be $(4.6-7.5) \times 10^{-12}$ $\mathrm{cm}^{-2} \mathrm{~s}^{-1}$, an $\mathscr{O}\left(10^{4}\right)$ above the expected DM emission.

\subsection{Dark Matter Clumps}

Cosmological N-body simulations predict existence of a large number of DM clumps distributed throughout the halos of galaxies. Not big enough to attract sufficient baryonic matter, these objects never started stellar formation process and as such should only be visible through gamma-ray photons from the DM annihilation [10]. The Fermi Satellite has detected a number of objects fitting the description of DM clumps: some of the Unassociated Fermi Objects (UFOs) have no counterparts at other wavelengths, show no variability and have a hard spectrum. However, due to limited energy range of Fermi, potential DM spectral features (like cutoff) have not be spotted. MAGIC, on the other hand, might be able to identify some of them as the DM sources.

During the few months in 2010/2011, two UFOs, best suitable for DM searches with MAGIC, have been observed [11]: 1FGL J2347.3+0710, for $8.3 \mathrm{~h}$ and 1FGL J0338.8+1313 for $10.7 \mathrm{~h}$. Neither case resulted in a clear detection, and integral flux ULs were calculated for energies above 100 $\mathrm{GeV}$, and are of order $3 \times 10^{-10}$ and $7 \times 10^{-11}$, respectively. The search for DM clump candidates in Fermi data continues to provide new observational targets for MAGIC.

\section{Conclusions}

MAGIC has been observing promising DM candidates since 2004. No detection has been claimed yet, and the calculated ULs are still far from constraining the mSUGRA parameter space. However, with the stereo system, prospects for the success are improved and continued efforts on dSphs, Galaxy Clusters and DM clumps are planned in the future.

\section{References}

[1] G. Bertone, D. Hooper and J. Silk, Phys. Rept., 405 (2005) [hep-ph/0404175]

[2] G. Bertone, Particle Dark Matter, Cambridge University Press, Cambridge 2010

[3] J. Aleksić et al., (2011) [astor-ph/11081477]

[4] H. P. Nilles, Phys. Rep., 110 (1984)

[5] J. Albert et al., ApJ Lett, 638 (2006) [astro-ph/0512469]

[6] J. Albert et al., ApJ, 679 (2008) [astro-ph/07112574]

[7] E. Aliu et al., ApJ, 697 (2009) [astrp-ph/08103561]

[8] J. Aleksić et al., JCAP 06 (2011) 035 [astro-ph/11030477]

[9] J. Aleksić et al., ApJ, 710 (2010) 634 [astro-ph/09093267]

[10] L. Pieri, G. Bertone and E. Branchini, MNRAS, 384 (2008) 1627 [astro-ph/0706.2101]

[11] D. Nieto et al., in proceedings of the ICRC (2011) [astro-ph/11095935] 